\title{
Boundedness of Solutions to Differential Equations of Fourth Order with Oscillatory Restoring and Forcing Terms
}

\author{
Cemil Tunç ${ }^{1}$ and Muzaffer Ateş ${ }^{2}$ \\ ${ }^{1}$ Department of Mathematics, Faculty of Sciences, Yüzüncü Yıl University, Van, Turkey \\ ${ }^{2}$ Department of Electrical \& Electronics Engineering, Yüzüncü Yıl University, Van, Turkey \\ Correspondence should be addressed to Cemil Tunç; cemtunc@yahoo.com
}

Received 25 June 2013; Accepted 17 August 2013

Academic Editor: Thabet Abdeljawad

Copyright @ 2013 C. Tunç and M. Ateş. This is an open access article distributed under the Creative Commons Attribution License, which permits unrestricted use, distribution, and reproduction in any medium, provided the original work is properly cited.

This paper deals with the boundedness of solutions to a nonlinear differential equation of fourth order. Using the Cauchy formula for the particular solution of nonhomogeneous differential equations with constant coefficients, we prove that the solution and its derivatives up to order three are bounded.

\section{Introduction}

In this paper, we study the boundedness of solutions to fourth-order nonlinear differential equation:

$$
x^{\prime \prime \prime \prime}+a x^{\prime \prime \prime}+f\left(x, x^{\prime}, x^{\prime \prime}\right)+g\left(x, x^{\prime}\right)+h(x)=p(t),
$$

where $x \in \Re, t \in[0, \infty), a>0, f, g, h, p$, and their first derivatives are continuous functions depending on the arguments shown. In addition, the functions $h$ and $p$ are oscillatory in the following sense: for each argument $u$, there exist numbers $\beta_{1}>\alpha_{1}>u>\alpha_{-1}>\beta_{-1}$ such that

$$
\varphi\left(\alpha_{1}\right)<0, \quad \varphi\left(\beta_{1}\right)>0, \quad \varphi\left(\alpha_{-1}\right)<0, \quad \varphi\left(\beta_{-1}\right)>0,
$$

where $\varphi$ is either $h(x)$ or $p(t), u$ is either $x$ or $t$, and all roots of the restoring term $h(x)$ are isolated.

It should be noted that there exist many papers dealing with boundedness of solutions to certain nonlinear differential equations of third and fourth order in the literature [1-15]. For nonlinear differential equations of fourth order, Afuwape and Adesina [1] used the frequency-domain approach to discuss the stability and periodicity of solutions, while Tunç and Tiryaki $[11,12]$ used intrinsic method to study the boundedness and stability of solutions. On the same time, Tunç [1315] used Lyapunov's second method to investigate the stability and boundedness properties of solutions of certain fourth order nonlinear differential equations. Further, other papers in this connection include those of Andres [2], Ogundare [6], and Omeike $[7,8]$, where the Cauchy formula was applied to evaluate the boundedness of solutions to certain third and fourth order nonlinear differential equations with oscillatory restoring and forcing terms.

The aim of this work is to extend and improve the previous studies and make some contributions to the literature since there are only a few papers on the boundedness of solutions of fourth order differential equations with oscillatory restoring and forcing terms (see [6-8]). It should be noted that the equation considered here, (1), includes and extends that of Ogundare [6] and Omeike $[7,8]$.

\section{Preliminary Results}

We need the following lemmas in the proof of our main result.

Lemma 1. One assumes that there exist positive constants $a, b$, $c, H$, and $P,\left(a^{2}>4 b\right)$ such that the following conditions hold for all $x \in R$ and $t \geq 0$ :

(i) $|h(x)| \leq H$,

(ii) $|p(t)| \leq P$,

(iii) $0<f\left(x, x^{\prime}, x^{\prime \prime}\right) / x^{\prime \prime} \leq b<\infty,\left(x^{\prime \prime} \neq 0\right), f\left(x, x^{\prime}, 0\right)=$ 0 ,

(iv) $0<g\left(x, x^{\prime}\right) / x^{\prime} \leq c<\infty,\left(x^{\prime} \neq 0\right), g(x, 0)=0$. 
Then, each solution $x(t)$ of (1) satisfies

$$
\limsup _{t \rightarrow \infty}\left|x^{\prime \prime \prime}(t)\right| \leq \frac{4(H+P)}{a}:=D^{\prime \prime \prime},
$$

provided that

$$
\begin{gathered}
\limsup _{t \rightarrow \infty}\left|x^{\prime}(t)\right| \leq \frac{H+P}{c}:=D^{\prime}, \\
\limsup _{t \rightarrow \infty}\left|x^{\prime \prime}(t)\right| \leq \frac{2(H+P)}{b}:=D^{\prime \prime} .
\end{gathered}
$$

Note that the constants $a, b$, and c satisfy the conditions ensuring that the auxiliary equation

$$
\lambda^{3}+a \lambda^{2}+b \lambda+c=0
$$

has negative real roots.

Proof. Substituting $z:=x^{\prime \prime \prime}$, we get from (1) that

$$
z^{\prime}+a z=p(t)-f\left(x, x^{\prime}, x^{\prime \prime}\right)-g\left(x, x^{\prime}\right)-h(x),
$$

with the solutions of the form

$$
\begin{aligned}
x^{\prime}(t)=z(t) & \\
=C e^{-a t}+\int_{T_{x}}^{t} e^{-a(t-\tau)} & {\left[p(\tau)-f\left(x(\tau), x^{\prime}(\tau), x^{\prime \prime}(\tau)\right)\right.} \\
& -g\left(x(\tau), x^{\prime}(\tau)\right) \\
& -h(x(\tau))] d \tau,
\end{aligned}
$$

where $C$ is an arbitrary constant and $T_{x}$ is a great enough number. Let us assume that the assumptions (4) and (5) hold. Thus, by the conditions of Lemma 1 , for $t \geq T_{x}$, we have not only

$$
\begin{aligned}
& \mid \int_{T_{x}}^{t} e^{-a(t-\tau)}\left[p(\tau)-f\left(x(\tau), x^{\prime}(\tau), x^{\prime \prime}(\tau)\right)\right. \\
& \left.\quad-g\left(x(\tau), x^{\prime}(\tau)\right)-h(x(\tau))\right] d \tau \mid \\
& \leq \int_{T_{x}}^{t}\left(|p(\tau)|+\left|\frac{f\left(x(\tau), x^{\prime}(\tau), x^{\prime \prime}(\tau)\right) \mid}{x^{\prime \prime}(\tau)}\right|\right. \\
& \left.\quad \times\left|x^{\prime \prime}(\tau)\right|\right) e^{-a(t-\tau)} d \tau \\
& +\int_{T_{x}}^{t}\left(\left|\frac{g\left(x(\tau), x^{\prime}(\tau)\right)}{x^{\prime}(\tau)}\right|\left|x^{\prime}(\tau)\right|\right. \\
& \quad+|h(x(\tau))|) e^{-a(t-\tau)} d \tau \\
& \leq \frac{4(H+P)}{a}\left(1-e^{-a\left(t-T_{x}\right)}\right), \\
& \quad \int_{T_{x}}^{t}\left(P+b\left|x^{\prime \prime}(\tau)\right|+c\left|x^{\prime}(\tau)\right|+H\right) e^{-a(t-\tau)} d \tau \\
& 4(H)
\end{aligned}
$$

but also

$$
\limsup _{t \rightarrow \infty}\left|x^{\prime \prime \prime}(t)\right| \leq \frac{4(H+P)}{a} .
$$

This completes the proof of Lemma 1.

Lemma 2. In addition to the assumptions of Lemma 1, one assumes that the following conditions hold:

(i) $\left|h^{\prime}(x)\right| \leq H^{\prime}$,

(ii) $\left|\int_{0}^{\infty} p(t) d t\right|<\infty$,

where $H^{\prime}$ is a suitable constant. Then, every bounded solution $x(t)$ of (1) either satisfies the relation

$$
\lim _{t \rightarrow \infty} x(t)=\bar{x}
$$

$$
\lim _{t \rightarrow \infty} x^{\prime}(t)=\lim _{t \rightarrow \infty} x^{\prime \prime}(t)=\lim _{t \rightarrow \infty} x^{\prime \prime \prime}(t)=0, \quad(h(\bar{x})=0)
$$

or there exists a root $\bar{x}$ of $h(x)$ such that $(x(t)-\bar{x})$ oscillates.

Proof. Let $x(t)$ be a fixed bounded solution of (1). Substituting this solution into (1) and integrating the result from $T_{x}$ to $t$ ( $T_{x}$-a great enough number), we obtain the following:

$$
\begin{aligned}
\int_{T_{x}}^{t} h(x(\tau)) d \tau \\
=-\int_{T_{x}}^{t} f\left(x(\tau), x^{\prime}(\tau), x^{\prime \prime}(\tau)\right) d \tau \\
\quad-\int_{T_{x}}^{t} g\left(x(\tau), x^{\prime}(\tau)\right) d \tau \\
\quad-x^{\prime \prime \prime}(t)+x^{\prime \prime \prime}\left(T_{x}\right)+a\left[-x^{\prime \prime}(t)+x^{\prime \prime}\left(T_{x}\right)\right] \\
\quad+\int_{T_{x}}^{t} p(\tau) d \tau(:=I(t)) .
\end{aligned}
$$

By noting the assumption (ii) of Lemma 1 and the boundedness of solution $x(t)$, it follows that there exists a constant $M_{x}$ for $t \geq T_{x}$ such that

$$
|I(t)| \leq M_{x}, \text { that is, }\left|\int_{T_{x}}^{t} h(x(\tau)) d \tau\right| \leq M_{x} .
$$

Now let us assume that $x(t)$ does not converge to any root $\bar{x}$ of $h(x)$, that is,

$$
\limsup _{t \rightarrow \infty}|x(t)-\bar{x}|>0
$$

and simultaneously,

$$
h(x(t)) \geq 0 \quad \text { or } \quad h(x(t)) \leq 0 \quad \text { for } t \geq T_{x} .
$$

Then,

$$
H(t):=\int_{T_{x}}^{t} h(x(\tau)) d \tau, \quad\left(t \geq T_{x}\right)
$$


evidently is a composed monotone function with a finite or infinite limit for $t \rightarrow \infty$.

Since (13) implies that the "divergent case" can be disregarded, then it follows from (15) that not only

$$
\lim _{t \rightarrow \infty} \int_{T_{x}}^{t}|h(x(\tau))| d \tau=\lim _{t \rightarrow \infty}\left|\int_{T_{x}}^{t} h(x(\tau)) d \tau\right| \leq M_{x}
$$

but also

$$
\liminf _{t \rightarrow \infty}|x(t)-\bar{x}|=0
$$

holds, because otherwise if (i.e., $\liminf _{t \rightarrow \infty}|x(t)-\bar{x}|>0$ ) (15) together with the fact that the roots of $h(x)$ are isolated would yield

$$
\liminf _{t \rightarrow \infty}|h(x(t))|=\liminf _{t \rightarrow \infty}|h(x(t))-h(\bar{x})|>0
$$

which is a contradiction to (17).

Thus, the estimates (14) and (18) imply that

$$
\limsup _{t \rightarrow \infty}|h(x(t))|=\limsup _{t \rightarrow \infty}|h(x(t))-h(\bar{x})|>0,
$$

and consequently, there exist such a sequence $\left\{t_{i}\right\} \geq T_{x}$ and a constant $\widetilde{H}>0$ such that (in what follows, $d(x, y)$ denotes the distance between $x$ and $y$ )

( $\alpha) \liminf _{t \rightarrow \infty \Rightarrow t_{i} \rightarrow \infty} d\left(t_{i}, t_{i-1}\right)>0$,

( $\beta)\left|h\left(x\left(t_{i}\right)\right)\right| \geq \widetilde{H}$

hold. Hence, the estimate

$$
M_{x} \geq \lim _{t \rightarrow \infty} \int_{t_{1}}^{t}|h(x(\tau))| d \tau=\sum_{i=2}^{\infty}\left|\int_{t_{i-1}}^{t_{i}} h(x(\tau))\right| d \tau
$$

implies that

$$
\lim _{t \rightarrow \infty \Rightarrow t_{i} \rightarrow \infty} \int_{t_{i-1}}^{t_{i}}|h(x(t))| d t=0
$$

or

$$
\begin{aligned}
H^{\prime} \limsup _{t \rightarrow \infty}\left|x^{\prime}(t)\right| & \geq \limsup _{t \rightarrow \infty}\left|\frac{d h(x(t))}{d x(t)} x^{\prime}(t)\right| \\
& =\limsup _{t \rightarrow \infty}\left|\frac{d h(x(t))}{d t}\right|=\infty .
\end{aligned}
$$

However, according to the assertion of Lemma 1, this case is impossible, and that is why $(x(t)-\bar{x})$ necessarily oscillates. The remaining part of Lemma 2 is followed from the assertion

$$
\begin{gathered}
x(t) \in C^{(n)}[0, \infty), \quad \lim _{t \rightarrow \infty}\left|x^{(n)}(t)\right|<\infty, \\
\lim _{t \rightarrow \infty}|x(t)|<\infty \Longrightarrow \lim _{t \rightarrow \infty} x^{(k)}(t)=0,
\end{gathered}
$$

where $n \geq 2$ is a natural number and $k=1, \ldots,(n-1)$. This completes the proof.
Lemma 3. In addition to the assumptions of Lemma 2, one assumes that the following conditions hold:

(i) $\left|p^{\prime}(t)\right| \leq P^{\prime}$,

(ii) $\lim \sup _{t \rightarrow \infty}|p(t)|>0$,

(iii) $\max \left\{\left|f_{x}\left(x, x^{\prime}, x^{\prime \prime}\right)\right|,\left|f_{x^{\prime}}\left(x, x^{\prime}, x^{\prime \prime}\right)\right|,\left|f_{x^{\prime \prime}}\left(x, x^{\prime}, x^{\prime \prime}\right)\right|\right\} \leq$ $b_{0}$,

(iv) $\max \left\{\left|g_{x}\left(x, x^{\prime}\right)\right|,\left|g_{x^{\prime}}\left(x, x^{\prime}\right)\right|\right\} \leq c_{0}$,

where $b_{0}, c_{0}$, and $P^{\prime}$ are suitable constants. Then, for every bounded solution $x(t)$ of (1), there exists a root $\bar{x}$ of $h(x)$ such that $(x(t)-\bar{x})$ oscillates.

Proof. If Lemma 3 does not hold, then according to Lemma 2, (11) holds and the fifth derivative of $x(t)$ satisfies

$$
\begin{aligned}
x^{(v)}(t)= & p^{\prime}(t)-a x^{\prime \prime \prime \prime}(t)-f_{x}\left(x, x^{\prime}, x^{\prime \prime}\right) x^{\prime}(t) \\
& -f_{x^{\prime}}\left(x, x^{\prime}, x^{\prime \prime}\right) x^{\prime \prime}(t)-f_{x^{\prime \prime}}\left(x, x^{\prime}, x^{\prime \prime}\right) x^{\prime \prime \prime}(t) \\
& -g_{x}\left(x, x^{\prime}\right) x^{\prime}(t)-g_{x^{\prime}}\left(x, x^{\prime}\right) x^{\prime \prime}(t) \\
& -h^{\prime}(x(t)) x^{\prime}(t) .
\end{aligned}
$$

Thus, by the assumptions of Lemmas 2 and 3, we have

$$
\begin{aligned}
\left|x^{(v)}(t)\right| \leq & P^{\prime}+a\left|x^{\prime \prime \prime \prime}(t)\right|+b_{0}\left|x^{\prime \prime \prime}(t)\right| \\
& +\left(b_{0}+c_{0}\right)\left|x^{\prime \prime}(t)\right|+\left(H^{\prime}+b_{0}+c_{0}\right)\left|x^{\prime}(t)\right| .
\end{aligned}
$$

Hence, by the boundedness of $x^{\prime}(t), x^{\prime \prime}(t), x^{\prime \prime \prime}(t)$, and $x^{\prime \prime \prime \prime}(t)$, it follows that there exists a constant $K$ such that

$$
\limsup _{t \rightarrow \infty}\left|x^{(v)}(t)\right| \leq K
$$

which according to (24), gives the following estimates:

$$
\begin{gathered}
\lim _{t \rightarrow \infty} x(t)=\bar{x} \Longrightarrow \lim _{t \rightarrow \infty} h(x(t))=h(\bar{x})=0, \\
\lim _{t \rightarrow \infty} x^{(j)}(t)=0, \quad j=1,2,3
\end{gathered}
$$

or

$$
\begin{aligned}
& \limsup _{t \rightarrow \infty}|p(t)| \\
& \quad=\limsup _{t \rightarrow \infty} \mid x^{\prime \prime \prime \prime}+a x^{\prime \prime \prime}+f\left(x, x^{\prime}, x^{\prime \prime}\right) \\
& +g\left(x, x^{\prime}\right)+h(x) \mid=0,
\end{aligned}
$$

which is a contradiction to $\limsup _{t \rightarrow \infty}|p(t)|>0$. This completes the proof of Lemma 3.

We now give the main result of this paper. 


\section{Main Result}

Theorem 4. One supposes that there exist positive constants $H, H^{\prime}, P, P^{\prime}, P_{0}$, and $R$ such that for $|x|>R$ and $t \geq 0$ the following conditions hold:

(i) $|h(x)| \leq H,\left|h^{\prime}(x)\right| \leq H^{\prime}$,

(ii) $0<f\left(x, x^{\prime}, x^{\prime \prime}\right) / x^{\prime \prime} \leq b<\infty,\left(x^{\prime \prime} \neq 0\right), f\left(x, x^{\prime}, 0\right)=$ 0 ,

(iii) $0<g\left(x, x^{\prime}\right) / x^{\prime} \leq c<\infty,\left(x^{\prime} \neq 0\right), g(x, 0)=0$

(iv) $|p(t)| \leq P,\left|p^{\prime}(t)\right| \leq P^{\prime},\left|\int_{0}^{t} p(\tau) d \tau\right| \leq P_{0}$, $\limsup _{t \rightarrow \infty}|p(t)|>0$,

(v) $\min \left[d\left(\bar{x}_{k}, \bar{x}_{k+1}\right), d\left(\bar{x}_{k}, \bar{x}_{k-1}\right)\right]>((H+P) / c)(4 / a+$ $2 a / b+b / c)+P_{0} / c$

where $\bar{x}_{k}$ are roots of $h(x)$ with $h^{\prime}\left(\bar{x}_{k}\right)>0$ and $\bar{x}_{k-1}, \bar{x}_{k+1}$ denote the couple of adjacent roots of $\bar{x}_{k}(k=0, \pm 2, \pm 4, \ldots)$. Then, all solutions $x(t)$ of (1) are bounded, and for each of them, there exists a root $\bar{x}$ of $h(x)$ such that $(x(t)-\bar{x})$ oscillates.

Proof. Let us assume, on the contrary, that $x(t)$ is an unbounded solution of (1), that is, $\limsup _{t \rightarrow \infty} x(t)=\infty$. Then, Lemma 1 implies the existence of a number $T_{0} \geq 0$ such that $t \geq T_{0}$,

$$
\begin{gathered}
\left|x^{\prime}(t)\right| \leq D^{\prime}+\varepsilon_{1}, \quad\left|x^{\prime \prime}(t)\right| \leq D^{\prime \prime}+\varepsilon_{2}, \\
\left|x^{\prime \prime \prime}(t)\right| \leq D^{\prime \prime \prime}+\varepsilon_{3},
\end{gathered}
$$

with $\varepsilon_{\mathrm{i}}>0,(i=1,2,3)$, small enough constants.

Let $T_{1} \geq T_{0}$ be the last point with $x\left(T_{1}\right)=\bar{x}_{k}(k$-even) and $T_{2}>T_{1}$ the first point with $x\left(T_{2}\right)=\bar{x}_{k+1}$. If we integrate (1) from $T_{1}$ to $t, T_{1} \leq t \leq T_{2}$, we come to

$$
\begin{aligned}
& x^{\prime \prime \prime}(t)-x^{\prime \prime \prime}\left(T_{1}\right)+a\left[x^{\prime \prime}(t)-x^{\prime \prime}\left(T_{1}\right)\right] \\
& +\int_{T_{1}}^{t} f\left(x(\tau), x^{\prime}(\tau), x^{\prime \prime}(\tau)\right) d \tau+\int_{T_{1}}^{t} g\left(x(\tau), x^{\prime}(\tau)\right) d \tau \\
& \quad+\int_{T_{1}}^{t} h(x(\tau)) d \tau=\int_{T_{1}}^{t} p(\tau) d \tau .
\end{aligned}
$$

Therefore, on replacing $\int_{T_{1}}^{t} f\left(x(\tau), x^{\prime}(\tau), x^{\prime \prime}(\tau)\right) d \tau$ and $\int_{T_{1}}^{t} g\left(x(\tau), x^{\prime}(\tau)\right) d \tau$ with $b\left[x^{\prime}(t)-x^{\prime}\left(T_{1}\right)\right]$ and $c\left[x(t)-x\left(T_{1}\right)\right]$, respectively, for $T_{1} \leq t \leq T_{2}$, we have $h(x(t)) \operatorname{sgn} x(t) \geq 0$. Multiplying (31) by sgn $x(t)$, we obtain

$$
|x(t)| \leq\left|x\left(T_{1}\right)\right|+\frac{2}{c}\left[D^{\prime \prime \prime}+a D^{\prime \prime}+b D^{\prime}+\frac{P_{0}}{2}\right]+\varepsilon,
$$

where $\varepsilon>0$ is an arbitrary small constant, a contradiction to $x\left(T_{2}\right)=\bar{x}_{k+1}$. The remaining part of the theorem follows from Lemma 3; therefore, we omit the details of the proof. The proof is complete.
Example 5. Consider the differential equation

$$
\begin{aligned}
& x^{\prime \prime \prime \prime}(t)+2 x^{\prime \prime \prime}(t)+\frac{5 x^{\prime \prime}(t)}{4\left[1+x^{2}(t)+x^{\prime 2}(t)\right]}+\frac{x^{\prime}(t)}{4+x^{2}(t)} \\
& +\frac{1}{100} \sin x(t)=\frac{1}{100} \cos t,
\end{aligned}
$$

where $a=2, b=5 / 4, c=1 / 4, h(x(t))=(1 / 100) \sin x(t)$, $p(t)=(1 / 100) \cos t$ with $\sin x(t)$ and $\cos t$, for $t \geq 0$, being oscillatory, and the equation

$$
\lambda^{3}+2 \lambda^{2}+\frac{5}{4} \lambda+\frac{1}{4}=0
$$

has negative real roots. A simple calculation (with the earlier notation) gives that $H=0.01, H^{\prime}=0.01, P=0.01, P^{\prime}=0.01$, and $P_{0}=0.01$. From the condition $(v)$ of the theorem, since $h(x(t))=(1 / 100) \sin x(t)$, then the roots of $h(x(t))$ are $\bar{x}_{k-1}=$ $(k-1) \pi, \bar{x}_{k}=k \pi$, and $\bar{x}_{k+1}=(k+1) \pi,(k=0, \pm 2, \pm 4, \ldots)$, where $\bar{x}_{k-1}$ and $\bar{x}_{k+1}$ are the couple of adjacent roots of $\bar{x}_{k}$.

Thus,

$$
\begin{gathered}
\min \left[d\left(\bar{x}_{k}, \bar{x}_{k+1}\right), d\left(\bar{x}_{k}, \bar{x}_{k-1}\right)\right]=\pi \\
\frac{H+P}{c}\left(\frac{4}{a}+\frac{2 a}{b}+\frac{b}{c}\right)+\frac{P_{0}}{c} \\
=4\left[(0.01+0.01)\left(2+\frac{16}{5}+5\right)+0.01\right]=0.856<1 .
\end{gathered}
$$

Since $\pi>1$, then all the conditions of the theorem are satisfied; thus, all solutions $x(t)$ of the above differential equation and their derivatives up to order three are bounded, and for each of them, there exists a root $\bar{x}$ of $h(x(t))$ such that $(x(t)-\bar{x})$ oscillates.

\section{References}

[1] A. U. Afuwape and O. A. Adesina, "Frequency-domain approach to stability and periodic solutions of certain fourthorder non-linear differential equations," Nonlinear Studies, vol. 12, no. 3, pp. 259-269, 2005.

[2] J. Andres, "Boundedness of solutions of the third order differential equation with oscillatory restoring and forcing terms," Czechoslovak Mathematical Journal, vol. 36, no. 1, pp. 1-6, 1986.

[3] J. Andres, "Boundedness results of solutions to the equation $x^{\prime \prime \prime}+a x^{\prime \prime}+g(x) x^{\prime}+h(x)=p(t)$ without the hypothesis $h(x)$ sgn $x \geq 0$ for $|x|>R$," Atti della Accademia Nazionale dei Lincei, Rendiconti. Classe di Scienze Fisiche, Matematiche e Naturali, vol. 80, no. 7-12, pp. 533-539, 1987.

[4] J. Andres, "Note to a certain third-order nonlinear differential equation related to the problem of Littlewood," in Proceedings of the 3rd International Seminar on Ordinary Differential Equations, vol. 23 of Fasciculi Mathematici, Poznań, Poland, 1990.

[5] W. A. Coppel, Stability and Asymptotic Behavior of Differential Equations, D. C. Heath, Boston, Mass, USA, 1965.

[6] B. S. Ogundare, "Boundedness of solutions to fourth order differential equations with oscillatory restoring and forcing terms," Electronic Journal of Differential Equations, vol. 2006, no. 6, 6 pages, 2006. 
[7] M. O. Omeike, "Boundedness of solutions to fourth-order differential equation with oscillatory restoring and forcing terms," Electronic Journal of Differential Equations, vol. 2007, no. 104, 8 pages, 2007.

[8] M. O. Omeike, "Boundedness of solutions of the fourth order differential equation with oscillatory restoring and forcing terms," Analele Stiintifice ale Universitatii "Alexandru Ioan Cuza" din Iasi, vol. 54, no. 1, pp. 187-195, 2008.

[9] R. Reissig, G. Sansone, and R. Conti, Non-Linear Differential Equations of Higher Order, Noordhoff International Publishing, Leyden, Mass, USA, 1974.

[10] K. E. Swick, "Boundedness and stability for a nonlinear third order differential equation," Atti della Accademia Nazionale dei Lincei, Rendiconti. Classe di Scienze Fisiche, Matematiche e Naturali, vol. 56, no. 6, pp. 859-865, 1974.

[11] C. Tunç and A. Tiryaki, "On the boundedness and the stability results for the solution of certain fourth order differential equations via the intrinsic method," Applied Mathematics and Mechanics, vol. 17, no. 11, pp. 979-988, 1996.

[12] A. Tiryaki and C. Tunç, "Boundedness and stability properties of solutions of certain fourth order differential equations via the intrinsic method," Analysis, vol. 16, no. 4, pp. 325-334, 1996.

[13] C. Tunç, "A note on the stability and boundedness results of solutions of certain fourth order differential equations," Applied Mathematics and Computation, vol. 155, no. 3, pp. 837-843, 2004.

[14] C. Tunç, "Some stability and boundedness results for the solutions of certain fourth order differential equations," Acta Universitatis Palackianae Olomucensis, vol. 44, pp. 161-171, 2005.

[15] C. Tunç, "An ultimate boundedness result for a certain system of fourth order nonlinear differential equations," in Differential Equations and Applications, vol. 5, pp. 127-135, Nova Science Publishers, New York, NY, USA, 2007. 


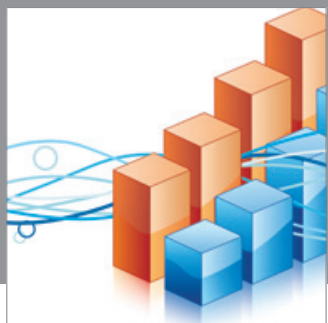

Advances in

Operations Research

mansans

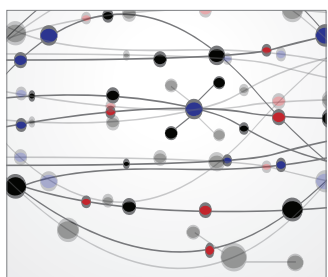

The Scientific World Journal
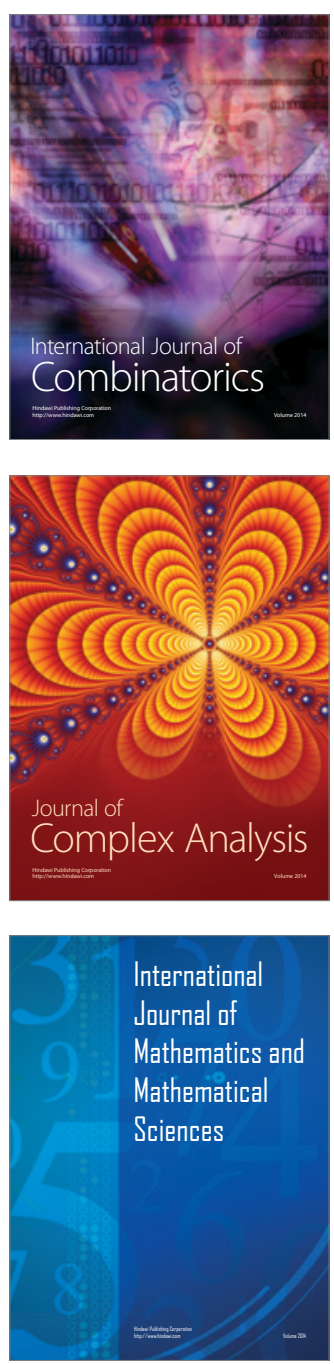
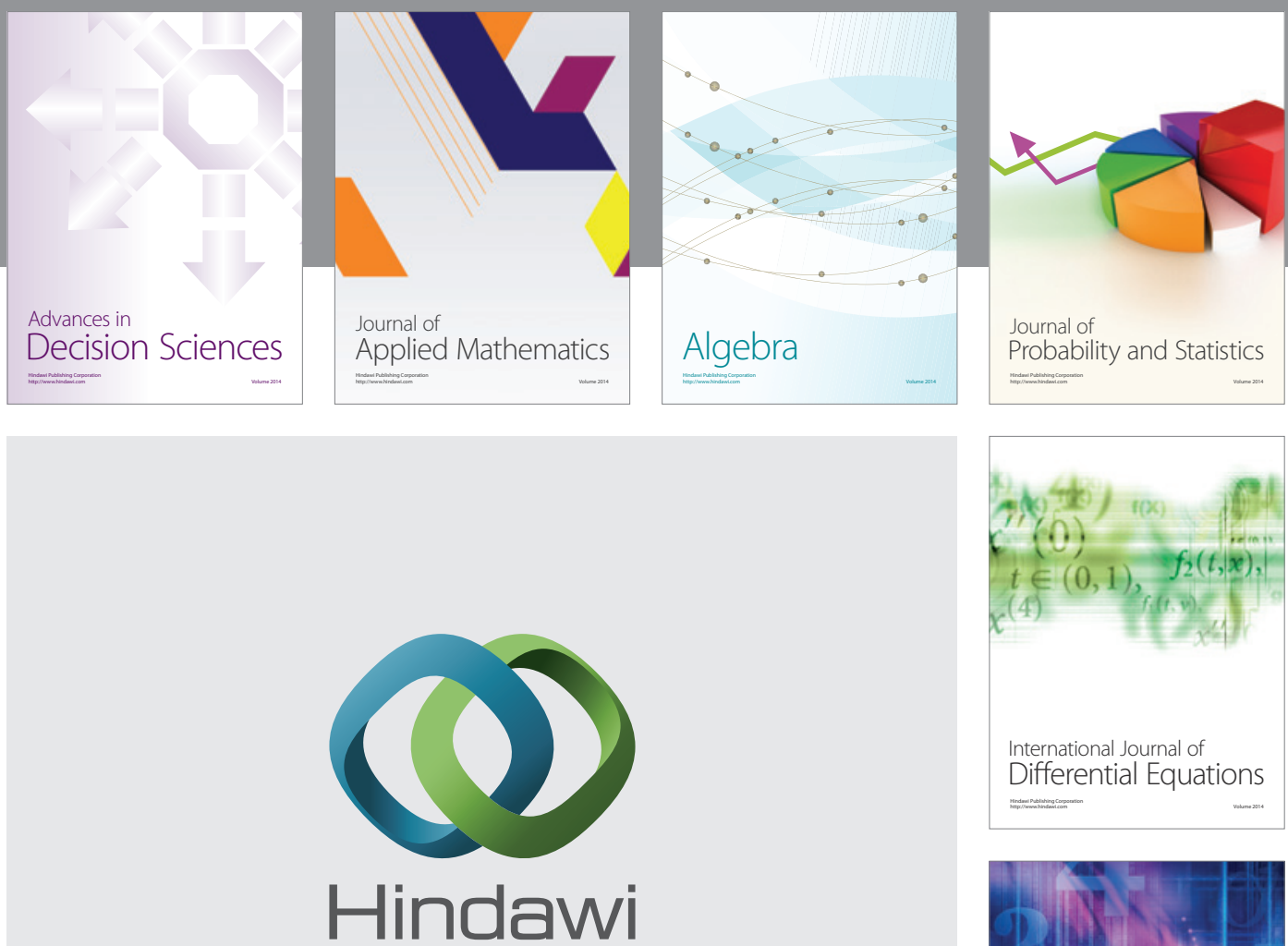

Submit your manuscripts at http://www.hindawi.com
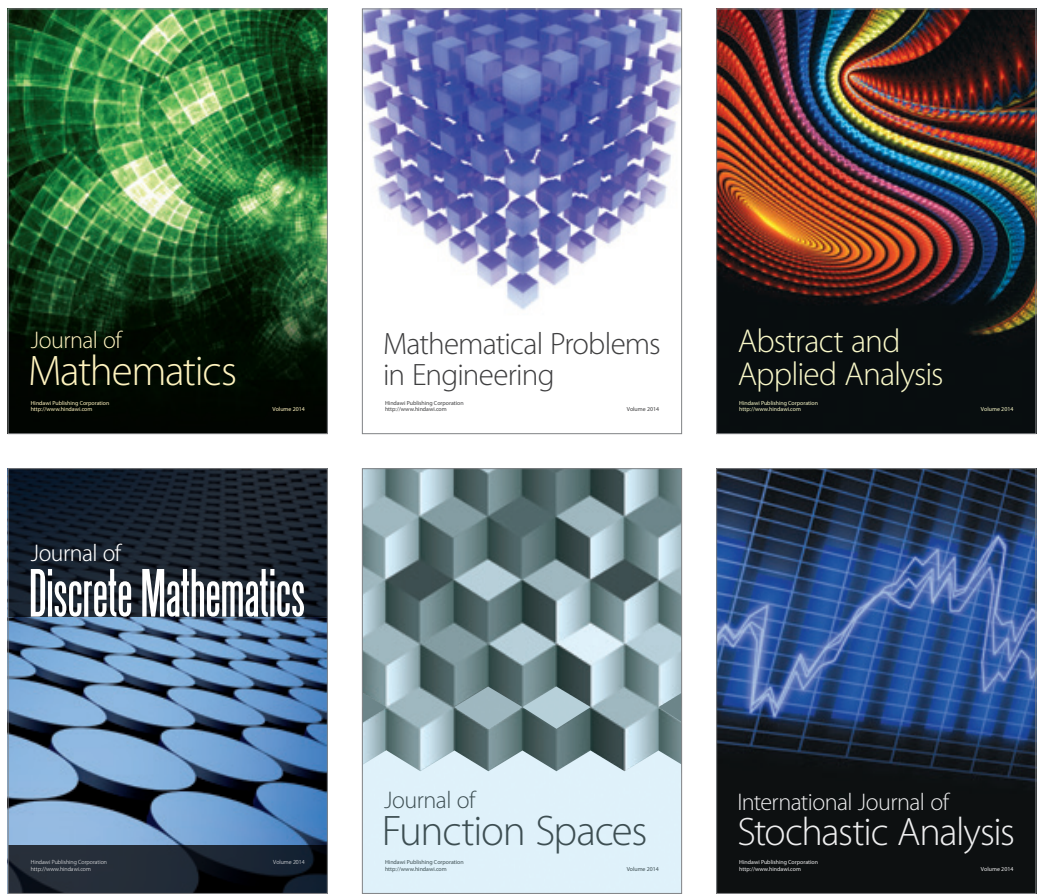

Journal of

Function Spaces

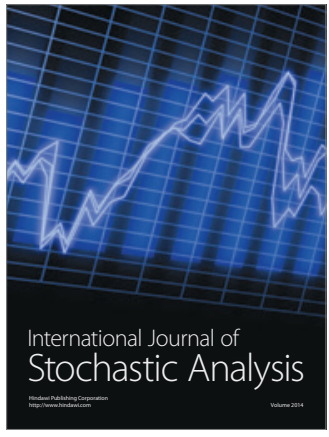

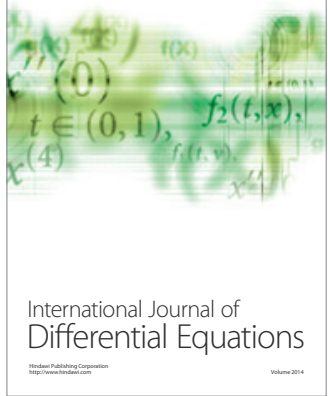
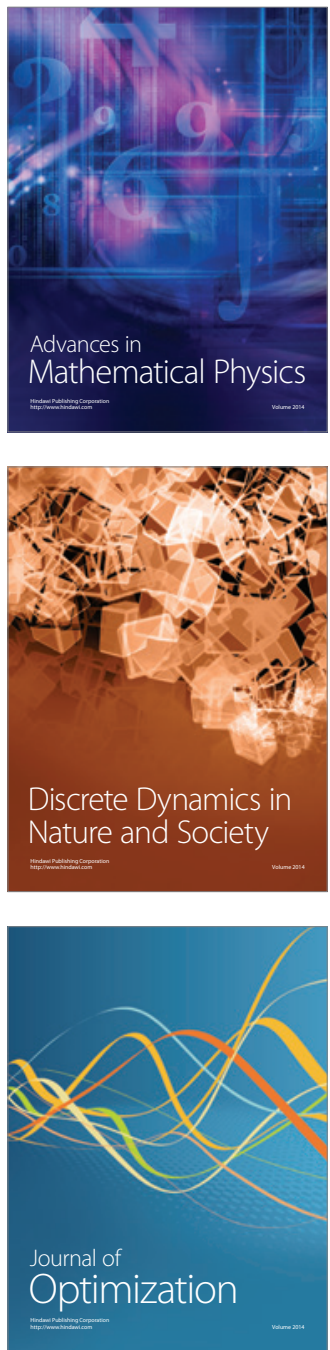